\title{
AMBIENT VIBRATION STUDIES FOR SYSTEM IDENTIFICATION OF TALL BUILDINGS
}

\author{
J. M. W. Brownjohn ${ }^{1}$ \\ ${ }^{1}$ Department of Civil \& Environmental Engineering, Nanyang Technological \\ University, \\ 50 Nanyang Avenue, Singapore 639798
}

\begin{abstract}
The performance of a building under wind and seismic loads depends on stiffness and mass distribution, and may be estimated using finite element codes. Experience has, however, shown that such finite element models often fail to predict the fundamental natural frequencies. Most of the time the frequencies will be underestimated, that is the building will turn out to be stiffer than anticipated, meaning the design would usually be conservative. On the other hand effects like torsional eccentricity and foundation compliance may not be correctly modelled, which could be less desirable.

A full understanding of linear performance under lateral loads can be obtained through experimental evaluation of the vibration modes though use of sensitive transducers (accelerometers) and appropriate data acquisition and modal analysis software. Traditionally a limited range of modal analysis procedures and software have been borrowed and applied to civil applications, but recent developments in system identification permitting modal analysis without measured input forces have been applied to a number of full-scale structures. The basis of some of these procedures are explained and the performance presented through case studies on two tall buildings in Singapore are reported here.

Experience shows that a quick and reliable estimation of mode shapes and frequencies can be obtained. Judgement of modal participation and damping ratios requires more detailed study yet the results are at least as convincing as existing frequency domain methods such as peak-picking.
\end{abstract}




\section{INTRODUCTION}

An increasingly important application for dynamic testing of civil infrastructure is to check for continuing structural integrity as buildings and bridges age and are subject to ever increasing loads, or after some specific damaging event such as an earthquake. At the same time, such tests are being correlated systematically with finite element analyses to improve or 'update' the analytical models from which structural parameters such as connection flexibility, Young's modulus or section geometric parameters can be identified (1). In civil structure applications such methods are only now beginning to be applied to buildings (2).

A second role for dynamic testing is to calibrate design codes and simulations of other structures. For example the development of empirical relationships for building natural frequency and damping ratio used in wind and seismic design codes (3) depends on some kind of dynamic testing at full-scale.

In each case, low level vibration testing to identify and track dynamic characteristics plays a central part in the investigation of structure performance. These modal parameters are:

1. natural frequency,

2. mode shape (vibration deformation pattern) and

3. damping ratio

\section{DYNAMIC TESTING}

A dynamic load applied to a structure can be considered as having components at a range of frequencies. The structure responds at all frequencies but will resonate when components coincide with natural frequencies of the structure. Through resonant amplification, an oscillating load can generate response one or two orders of magnitude higher than a static load of the same amplitude. Conversely very small loads can generate measurable response, for example office towers and masts can be excited enough by one person shifting his body weight (4). For long span floors and footbridges the dynamic loading of one pedestrian can lead to significant deflections (5).

Vibration transducers are traditionally far more sensitive to small movements than displacement transducers, for example an accelerometer that can resolve an acceleration of $10^{-5} \mathrm{~m} / \mathrm{sec}^{2}(1 \mu \mathrm{g})$ can sense vibrations of $10^{-7} \mathrm{~m}$ at a frequency of $1 \mathrm{~Hz}$, a typical fundamental mode frequency for a tall building. The net result is that very small dynamic loads can generate signals that are relatively easy to measure yet can provide detailed structural information.

There are two forms of dynamic test on civil structures, forced vibration test (FVT) and ambient vibration test (AVT) (6). In some cases (7) it may be possible to force or artificially excite vibrations while at the same time measuring the applied load due to an electro-dynamic, hydraulic or mechanical shaker. Traditionally, for buildings rotating eccentric mass (REM) shakers (8) are used for stepped-sine testing, one frequency at a time. In rare cases shakers driven by hydraulic or electro-dynamic actuators (9) have been used to excite a building with a broadband signal. The motivation for the considerable 
trouble of FVT with such machines has been that traditional modal analysis procedures such as circle fit (10) could be used, often with greater accuracy in identifying some of the parameters, particularly the damping ratios.

Because of the logistics and large machinery associated with FVT, in all but a few exceptional cases, AVT is a simple and preferable choice. A set of accelerometers may be placed on the structure to measure systematically the vibrations resulting from the wind, traffic or people; these are so-called ambient vibrations and the AVT has been used for at least half a century (11). In some cases it is the only possibility, for example very tall buildings or huge suspension bridges whose very low fundamental frequencies put them beyond the effective operating envelope of all but the very largest FVT shakers (7).

\section{MODAL ANALYSIS OF CIVIL STRUCTURES WITH RESPONSE-ONLY AVT DATA}

Mechanical and aerospace engineers use modal analysis software (10) specifically designed for their single input multiple output multiple input multiple output tests (12). Civil structure dynamic testing rarely uses more than single input and in the case of 'response only' measurements conventional modal analysis procedures cannot be used. The situation has changed within the last decade through development of a number of system identification procedures. It is now relatively easy to obtain a quick and reasonable estimate of modal parameters from examination of response to random loading that has a power spectrum that is approximately flat.

There are still some issues on the reliability of modal parameters such as damping derived from AVT data using these procedures. However, results obtained from testing a number of structures in Singapore have demonstrated that reliable information about dynamic behaviour can be obtained from low cost and non-invasive AVT.

The basis and performance of modern 'response only' modal analysis are examined with reference to two tall buildings tested using these methods.

\section{Office Tower 1}

The studied building in Singapore is Republic Plaza (Fig. 1). The building, which is thoroughly described in previous publications (20), was completed in early 1996 and has one basement level and 65 storeys rising to $280 \mathrm{~m}$. A reinforced concrete core wall resists lateral load while tubular concrete-filed steel columns with steel framing systems at every storey transfer vertical load to the very rigid foundation. According to the floor plan of the core wall at level (storey) 65, Fig. 2 , there are obvious axes of symmetry.

\section{Office Tower 2}

The 31-storey JTC (Jurong Town Corporation) Summit building (Fig. 3) was completed in 2000. It has three basement levels and 31 storeys, rising to $141 \mathrm{~m}$, and is adjoined by a four storey podium block. The entire structure weighs approximately $500 \mathrm{MN}$, is founded on bored piles and comprises a reinforced concrete moment resisting frame with a central core to provide resistance to 
lateral loads. According to the floor plan (Fig. 4) the building has no axes of symmetry.

\section{THEORETICAL BASIS OF AVT MODAL ANALYSIS}

Traditionally AVT has used peak-picking, which works on the frequency domain ratio of response signals through consideration of a common relationship with an unknown input. Specifically, for a specific (angular) frequency $\omega$ the ratio of acceleration response $\ddot{X}_{j}(\omega)$ at level $j$ to force input $P_{k}(\omega)$ at level $k$ on a building is defined as the inertance form of the frequency response function (FRF):

$$
H_{i j}(\omega) \equiv \ddot{X}_{j}(\omega) / P_{k}(\omega) \text {. }
$$

By considering the equations of motion it can be shown (12) that

$$
H_{j k}(\omega)=\sum_{r=1}^{N} H_{r}{ }^{r} \phi_{j}{ }^{r} \phi_{k}
$$

where

$$
H_{r}(\omega)=\frac{-\omega^{2}}{-\omega^{2}+\omega_{r}^{2}+2 i \zeta_{r} \omega_{r}}
$$

is the dynamic amplification factor and ${ }^{~} \phi_{j}{ }^{r} \phi_{k}$ is the modal constant containing information about mode shapes ${ }^{r} \phi$ and mass distribution for one of $N$ possible modes $r$ having natural frequency and damping ratio $\omega_{r}, \zeta_{r}$. If one row or column of the FRF matrix is obtained by FVT, the modal parameters can be identified (10).

For AVT of a building it is assumed that unmeasured forces $P_{k}(\omega)$ are applied simultaneously at all $m$ possible levels $k$, so the response at level $j$ is

$$
\ddot{X}_{j}(\omega)=\sum_{k} P_{k}(\omega) \sum_{r=1}^{N} H_{r}(\omega)^{r} \phi_{j}{ }^{r} \phi_{k}=\sum_{k} \sum_{r=1}^{N} P_{k}(\omega) H_{r}(\omega)^{r} \phi_{j}{ }^{r} \phi_{k} \text {. }
$$

Also it has to be assumed that the input force is not a function of frequency but has a spectrum with constant (stationary) mean value at each frequency. This is not quite true for wind, and several semi-empirical expressions for power spectrum of wind pressures are available (13). Even so, compared to the fast changing $H_{r}(\omega)$ the square root power spectrum of wind force per storey is only a weak function of frequency. The net effect is obtained by summing over the levels $k$ while taking into account the lack of coherence between loading at different levels, leading to an approximate distributed effect $C$ and response depending on level $j$ i.e.

$$
\ddot{X}_{j}(\omega)=C \sum_{r=1}^{n} H_{r}(\omega)^{r} \phi_{j} .
$$


The spectral approach to predicting dynamic response due to wind is described in detail in other references $(14,15)$. For excitation by ground motion similar analysis can be used except that the effective forces at each storey are coherent and proportional to ground motion.

From measured time series of acceleration response $\ddot{x}_{j}(t)$, the scalar discrete Fourier transforms (FFTs) of $m$ channels of $2 n$ response data are formed into a $n \times m$ vector of complex FFTs $\ddot{\mathbf{X}}(\omega)$. From all $m^{2}$ combinations is constructed a $n \times m \times m$ matrix of cross-power spectra. In practice an average is usually made over a recording divided into $M$ frames of $2 n$ samples using the Welch method (16), where windowing and overlapping may be applied. Hence

$$
\overline{\mathbf{S}}_{x x}(\omega)=E\left(\ddot{\mathbf{X}}(\omega) \ddot{\mathbf{X}}^{H}(\omega)\right)
$$

is the experimentally derived matrix of cross-power spectra containing at row $p$ and column $q$ the complex scalar cross-power:

$$
S_{q p} \equiv \bar{S}_{X_{q} X_{p}}=E\left(\ddot{X}_{p}(\omega) \ddot{X}_{q}^{*}(\omega)\right)
$$

The superscripts ${ }^{*}$ and ${ }^{H}$ denote complex conjugate and complex conjugate matrix transpose.

\section{Peak picking and operating deflection shapes (ODS)}

Usually the mode shape is estimated by varying $p$ while keeping a reference $q$ constant and reading off the values down a column of the cross-power matrix at a resonant frequency identified as a peak in the spectrum. These values contain phase and amplitude information and are normalised to a value of 1 with angle zero by dividing by the auto-power of the chosen reference signal. This is called 'peak picking' and is equivalent to evaluating the 'transfer function' between measured response positions $p$ and $q$ :

$$
\frac{S_{q p}(\omega)}{S_{q q}(\omega)}=\frac{E\left(\ddot{X}_{p}(\omega) \ddot{X}_{q}^{*}(\omega)\right)}{E\left(\ddot{X}_{q}(\omega) \ddot{X}_{q}^{*}(\omega)\right)}=E\left(\frac{\ddot{X}_{p}\left(\omega_{r_{o}}\right)}{\ddot{X}_{q}\left(\omega_{r_{o}}\right)}\right)
$$

At a specific natural frequency $\omega_{r_{o}}$ the contribution of mode $r_{o}$ to response dominates over all the other modes hence:

$$
E\left(\frac{\ddot{X}_{p}\left(\omega_{r_{o}}\right)}{\ddot{X}_{q}\left(\omega_{r_{o}}\right)}\right)=E\left(\frac{C \sum_{r=1}^{N} H_{r}\left(\omega_{r_{o}}\right)^{{ }^{\prime} \phi_{p}}}{C \sum_{r=1}^{N} H_{r}\left(\omega_{r_{o}}\right)^{r \phi_{q}}}\right) \approx \frac{C H_{r_{o}}{ }^{{ }{ } \phi_{p}}}{C H_{r_{o}}{ }^{{ }}{ } \phi_{q}}
$$

hence as all but $H_{r_{o}}$ are negligible at $\omega_{r_{o}}$,

$$
\frac{S_{q p}\left(\omega_{r_{o}}\right)}{S_{q q}\left(\omega_{r_{o}}\right)} \approx \frac{{ }^{{ }} \phi_{p}}{{ }^{{ }^{\circ}} \phi_{q}} \text {. }
$$


The ratio of cross-powers recovers the approximate ratio of modal ordinates between two locations. If the denominator is a constant an 'operating deflection shape' (ODS) is recovered, which is an estimate of mode shape. Further, the auto-power spectrum $S_{q q}(\omega)$ around the resonance can be used for estimation of frequency and damping ratio through curve-fitting. Spectral estimates used in equation 9) are subject to variance errors in inverse proportion to the number of averages used in the Welch procedure (100 averages are required for $1 \%$ coefficient of variation) and at least part of this variance carries over to mode shapes. Damping, and to a lesser extent frequency, are subject to bias and variance errors (17) according to parameters such as type of window used, number of averages and width of the resonant peak in relation to frequency resolution. For civil structures with low damping, very long periods of measurement are required and it is or needs to be assumed that the loading spectrum is Gaussian and the modal parameters are stationary (constant) for the duration.

\section{NExT: Natural Excitation Technique}

Procedures are now available to improve the accuracy and ease of AVT data analysis and the ability to discriminate close modes. One valuable contribution is the Natural Excitation Technique (NExT)(18) that can obtain a set of frequencies, damping ratios and true mode shapes. NExT is a kind of generic procedure, a similar procedure is the Subspace Identification (SSI).

NExT may use the Eigensystem Realisation Algorithm (ERA) $(19,20)$ applied to a set of impulse response functions which can be obtained either through crosscovariance functions of the original time series or the inverse Fourier transforms of their frequency domain equivalent, the cross-power spectra. In the applications described here the procedure is termed NExT/ERA.

A discretised structural system is represented as having mass, stiffness and damping matrices $\mathbf{M}, \mathbf{C}$ and $\mathbf{K}$. For a 'stick model' of a building having one lumped mass at each floor and one spring element (column) between floors, the storey or level displacements form a vector $\mathbf{x}$. The equations of motion are then written as

$$
\mathbf{M} \ddot{\mathbf{x}}+\mathbf{C} \dot{\mathbf{x}}+\mathbf{K x}=\mathbf{p} \text {. }
$$

These are transformed to the 'state space' form of first order equations

$$
\dot{\mathbf{z}}=\mathbf{A z}+\mathbf{B p}
$$

where $\mathbf{A}=\left[\begin{array}{cc}\mathbf{M}^{-1} \mathbf{C} & \mathbf{M}^{-1} \mathbf{K} \\ \mathbf{I} & \mathbf{0}\end{array}\right] \mathbf{z}=\left[\begin{array}{c}\dot{\mathbf{x}} \\ \mathbf{x}\end{array}\right]$ and $\mathbf{B}=\left[\begin{array}{c}\mathbf{M}^{-1} \\ \mathbf{0}\end{array}\right]$.

The frequencies and damping ratios of the MKC system of equation (11) appear as conjugate pairs of eigenvalues of $\mathbf{A}$ :

$\lambda_{\mathrm{A}}=-\xi \omega \pm i \omega \sqrt{1-\xi^{2}}$ 
and the eigenvectors of $\mathbf{A}$ are the eigenvectors of equation (11) with zero input and either zero or proportional damping. Hence once a form of $\mathbf{A}$ representing a discretised modal model of the true structure can be found from test data, the modal parameters can be 'realised'. ERA recovers a candidate A from impulse response functions (IRFs) that represent the multi-mode behaviour described by equations 10) and 11). The IRFs can be shown (18) to be equivalent (except for a common scale factor) and contain the same information when recovered from:

a) Directly measured impulse response functions

b) Inverse FFTs of frequency response functions such as given in equation 1)

c) Cross-covariance functions of random responses to a common (Gaussian) excitation

d) Inverse FFTs of cross-power spectra such as given in equation 6) for common (Gaussian) excitation channels

Fig. 5 shows, for Office Tower 1 , the time series of basement (channel 1) and level 65 (channel 3) response resulting from weak ground motions due to a tremor some $600 \mathrm{~km}$ distant. The spectrograms show the broadband character of the ground motion and resonant amplification at the upper floors. Fig. 6 shows cross-covariance functions $R$ computed in time domain between the two signals; the values of $R_{11}, R_{13}, R_{31}$ and $R_{33}$ at each time step $i$ form a square matrix or $2 \times 2$ 'block' $\mathbf{X}_{i}$. The functions of Fig. 6 are obtained directly from the short sequence of the data of Fig. 5 and represent case c) above. Equivalent plots could be obtained as in case d) by inverse FFT of cross-power spectra either from a single measurement or from averaging over many records (e.g. obtained during strong winds).

Fig. 7 shows the transmissibility function between channel 1 and channel 3 for the data of Fig. 5, in frequency and time domains. This is case b) above as transmissibility is defined in equation 1) replacing the excitation $P_{k}(\omega)$ with ground acceleration $\ddot{X}_{g}(\omega)$. In this case there is only one trace and a scalar impulse response function $h_{13}$, whose values at time step $i$ can also be represented as $\mathbf{X}_{i}$.

The examples are for scalar or square $\mathbf{X}_{i}$. A third and more usual case is where IRFs, FRFs, cross-covariance functions or cross-power spectra are formed between a group of $n$ signals and a common reference; in this case $\mathbf{X}_{i}$ is a $n \times 1$ column vector.

\section{ERA: Eigensystem Realisation Algorithm}

With $\mathbf{X}_{i}$ representing as a scalar, vector or matrix the cross-covariance or impulse response function (IRF) at the th time sample, a matrix is constructed by stacking $\mathbf{X}_{i}$ into a matrix: 


$$
\mathbf{H}(0)=\left[\begin{array}{cccc}
\mathbf{X}_{0} & \mathbf{X}_{1} & \cdot & \mathbf{X}_{a} \\
\mathbf{X}_{1} & \mathbf{X}_{2} & \cdot & \mathbf{X}_{a+1} \\
\cdot & \cdot & \cdot & \cdot \\
\mathbf{X}_{b} & \mathbf{X}_{b+1} & \cdot & \mathbf{X}_{a+b}
\end{array}\right]
$$

This is a Hankel matrix 'at time $0^{\prime}$ and the sequence of $\mathbf{X}_{i}$ are known as the Markov parameters. As $\mathbf{H}(0)$ contains the it also contains information about the mode shapes, frequencies and decay rates. As more rows and columns are added the rank grows until, in theory, it reaches a limit according to number of vibration modes contributing. A minimum rank form of $\mathbf{H}$ is reconstructed and a state matrix $\mathbf{A}$ is recovered from it. ERA is described very thoroughly elsewhere (21) and a summary is given here.

The $a, b$ in $\mathbf{H}(0)$ are parameters relating to the number of samples used, and it is a balance to decide on an appropriate size of $\mathbf{H}(0)$ to capture the long frequency components of the impulse response functions without losing those that decay quickly.

The singular value decomposition (SVD) of the Hankel matrix is carried out to find the system order:

$$
\mathbf{U S V}^{\prime}=S V D[\mathbf{H}(0)]
$$

The effective rank of $\mathbf{H}(0)$ is obtained from the largest $N$ singular values, according to choice or some numerical criteria, and corresponding sub-vectors and matrices $\mathbf{S}_{N}, \mathbf{V}_{N}, \mathbf{U}_{N}$ are used to regenerate the state matrix $\mathbf{A}$ :

$$
\mathbf{A}=\mathbf{S}_{N}^{-1} \times \mathbf{U}_{N}^{\prime} \times \mathbf{H}(1) \times \mathbf{V}_{N} \times \mathbf{S}_{N}^{-1}
$$

The eigenvalues of $\mathbf{A}$ provide natural frequencies and damping ratios (by equation 12) and eigenvectors correspond to those of the structure tested.

\section{Improved Peak Picking}

A novel and very simple procedure that represents an improvement on peak picking has been proposed (22). The singular value decomposition (SVD) of the cross-power spectra matrix is performed at each frequency line (bin) $i$ :

$$
\left[\mathbf{U S V}^{\prime}\right]_{i}=\left[\mathbf{S}_{\mathbf{x x}}\right]_{i}
$$

The first singular value represents the strength of the dominating vibration mode at each bin and the singular vectors at each bin contain the ODS. The remaining singular values will contain either noise or weaker modes 'hidden' under a strong dominating mode. Hence at least one advantage of the method is to clean up an auto power spectrum and remove the need to choose which of the channel to use for curve fitting to recover frequencies and damping ratios.

Fig. 8 shows auto power spectra at level 65 of Office Tower 1 with frequency resolution of $5 \mathrm{mHz}$ and 38 averages; fine resolution separates the peaks well 
enough due to toe low damping. Fig. 9 shows the first two singular values; the first represents both modes equally while the second obviously represents an artificial mode. The ODS in the first singular vector is the same as that identified by other methods (NEXT/ERA and peak-picking).

It can be shown (23) that where the second mode is weak the second singular value does recover the 'hidden mode' subject to some conditions on the orthogonality of the mode shapes. A form of this method termed 'Frequency Domain Decomposition' is implemented in commercially available software but the method as applied here is termed 'Improved Peak Picking (IPP). 


\section{APPLICATION OF AVT PROCEDURE}

Office Towers 1 and 2 have been studied via AVT. Results of an elaborate exercise on Office Tower 1 using a form of peak picking have been reported previously (24) and a more recent modal analysis exercise using signals from a long term monitoring system is reported here. No prior data were available for Office Tower 2 and NExT/ERA and IPP were used to obtain a comprehensive modal characterisation.

\section{AVT of Office Tower 1}

This was tested just after completion at the end of 1995. In the original modal survey a set of four high-resolution force-balance accelerometers was used; one was kept as a reference at the highest habitable point in the building; the mechanical equipment floor at level 65 . The other three accelerometers were moved to levels from ground to 66 in stages and according to the available length of signal cable (the building reaches a height of $280 \mathrm{~m}$ ). A set of modes was identified (24) and frequencies for translational modes are shown in Table 1.

One problem with the original measurements was discriminating the response between the two directions in a pair of modes at approximately $0.2 \mathrm{~Hz}$. In the peak picking procedure the closeness of the modes and limited frequency resolution meant that for measurements that always contained both signals a pure single mode response could not be identified. Also, while the building appears to be symmetric, the arrangement of the core structure leads to eigenvectors not coinciding with natural axes so signals in $A$ and $B$ directions contain response in two fundamental modes. Directions of the two fundamental modes were originally identified by comparing auto-power spectra from $A$ and $B$ directions and are given in Table 1.

Since 1996 signals from pairs of accelerometers installed in the basement and level 65 have been recorded during 'significant event' e.g. tremors and strong winds. The sample rate is fixed at $7.5 \mathrm{~Hz}$ to capture signals in the range $0-3 \mathrm{~Hz}$. Fig. 10 shows, on a logarithmic scale, the auto-power spectra of acceleration, the diagonals of the cross-power matrix, obtained from one series of measurements in two orthogonal directions $A$ and $B$ coinciding with the "natural axes of the building shown in Fig. 2. The cross-powers for these data were analysed using NExT/ERA, allowing to show directly the vibration axes in Fig. 11. The frequencies given in Fig. 11 are slightly lower than those in Table 1 since the data of Fig. 10 were obtained from recent measurements after the building was occupied and considerable extra mass had been added. Also, two torsional modes are included in the sequence.

\section{AVT of Office Tower 2}

Students participating in a final year project in November/December 2000 planned and executed a program of measurements a larger set of 16 accelerometers. These were deployed in a sequence to measure accelerations in $\mathrm{X}$ and $\mathrm{Y}$ directions throughout the two stairwells, at each storey including the basement and roof, corresponding to 138 'degrees of freedom'. For each 
measurement a reference pair of accelerometers was maintained measuring in $\mathrm{X}$ and $\mathrm{Y}$ directions at storey 31 .

Fig. 12 shows the measurement grid with measurement points on two vertical lines up the two stairwells. Nodes 1 and 36 correspond to corresponding to third basement (B3), nodes 35 and 70 are at storey 32 (the roof). Nodes 1-35 are in stairwell 1, closest to the curved corner of the building and reference accelerometers were located at node 34. After an assumption of floor slabs behaving as rigid bodies was checked experimentally, the horizontal motion of the complete building could be derived from measurements at the given grid points via kinematic relationships.

The 14 roving accelerometers (rovers) were deployed measuring first in $\mathrm{Y}$ direction in stairwell 1 at even storey levels, then in X-direction. Rovers were then moved to odd storey levels to measure in each direction and then measurements were made to fill in 'gaps' including poor signals. The 14 rovers were moved to stairwell 2 and the process was repeated, providing a comprehensive set of measurements in each direction at each storey level from third basement through to roof level. In a final series of measurements all 16 accelerometers were deployed simultaneously measuring in Y-direction throughout both stairwells. For all recordings the sample rate was fixed at $50 \mathrm{~Hz}$.

Due to very weak (i.e. usually totally absent) wind excitation, unidentified mechanical and electrical signal interference effects and occasional instrumentation problems, many measurements were repeated, but even so the best data from certain locations are still very noisy.

Fig. 13 shows a good example of auto power spectra from the reference accelerometers. As it was relatively windy during this measurement, modes are sharp, well defined and have low damping. In fact modes at $5.38 \mathrm{~Hz}$ and $7.44 \mathrm{~Hz}$ seem suspiciously sharp suggesting a mechanical vibration source. For the reasons given above, few signals were as clear as these, but by rejecting redundant data of poor quality and piecing together the remains, a clear set of vibration modes was identified.

NExT/ERA was used on the inverse FFT of a single column vector of crosspowers obtained from stacking cross-powers of a selection of measurements or a complete set, referenced to one or other of the two 'fixed' accelerometers. For the full set of measurements, which included duplication, each $\mathbf{X}_{i}$ added to $\mathbf{H}$ was a vector of over 200 points. The optimal size of $\mathbf{H}$ was found to be $a=300$, $b=10$.

The finally identified set of 12 modes is presented in Table 2 . The frequency and damping values are best estimates obtained from the best of the data. Due to lack of symmetry the vibration modes do not fall into neat patterns of modes which can be described as lateral (X-direction), longitudinal (Y-direction) or torsional (rotating about a geometric centroid), but the predominant form of motion is indicated in Table 2. 
Figs. 14-16 attempt to illustrate the mode shapes. Figure 14 shows lateral $(Y)$ components of modes recovered from measurements only in Y-direction. The final set of measurements during relatively strong winds but using a coarse grid of points measuring only in $\mathrm{Y}$ direction confirms this pattern. Fig. 15 shows elevation of modes recovered from measurements only in X-direction. Modes not recovered in these measurements (and therefore not participating in that direction) are not shown. Figure 16 shows, in plan, the components of mode shapes at the roof thus providing the visual link between $X$ and $Y$ components of the modes.

Mode shapes in Figs. 14-16 are shown without any smoothing of points or curve-fitting. Even allowing for anomalies in some of the measurement points the complexity of the modes is clear. Figure 16 shows that no single mode is either pure lateral $(\mathrm{Y})$, longitudinal $(\mathrm{X})$ or torsional response.

\section{Performance of NExT/ERA}

The NExT/ERA procedure, or similar forms, is available in commercial packages as well as in shareware (25), and is now quite widely used. It clearly has benefits which include the applicability to ambient or forced vibration data described in both frequency and time domain. As originally done for Office Tower 1, data from Office Tower 2 could have been analysed with peak picking from cross-powers. In fact part of the student exercise employed an implementation of IPP to recover a set of the lower modes, but only the first six could be reliably recovered. NExT/ERA was able to identify an extended set of modes and provide actual mode shapes (not ODS). The modes were well separated for this building so ODS would be acceptable, but the close fundamental modes of Office Tower 1 provided a real test.

Most researchers engaged in full-scale testing can tell if a vibration mode is realistic. A realistic mode has a shape that fits into a pattern of mode shapes and frequencies and does not have excessive or uncharacteristically low damping. There will be a good 'fit' of the mode response to an analytical form in either time or frequency domain. Such modes will not normally be strongly complex among real modes having mode shapes ordinates in phase or out of phase (i.e. at $0^{\circ}$ or $180^{\circ}$ within a reasonable tolerance).

Pick picking (also IPP) normally limits identification to those modes having strong and recurring peaks in auto-power spectrum, while NExT/ERA has no such restriction. Depending on the size and shape of the Hankel matrix via the shape of $\mathbf{X}_{i}$ as well as $a$ and $b$, and the number $N$ of retained singular vectors used to construct the state matrix, the procedure just returns a pre-determined number of modal parameters leaving the user to sort out which are realistic. This usually means judging against prior knowledge e.g. from more conventional modal analysis. Without such physical engineering judgement, the user has to judge which modes are fictitious (mathematical artifices or noise) based on numerical indicators and convergence studies. 
Converge plots (26) are commonly used to demonstrate the effect of changing $N$. As $N$ increase up to a point all the 'known' modes appear more convincingly, and beyond that point artificial modes appear.

Fig. 17 plots the modes identified from the cross-power spectra of Fig. 8 as more $2 \times 2$ blocks $\mathbf{X}_{i}$ are stacked into $\mathbf{H}$, so that $a$ and $b$ of equation 13) are simultaneously incremented to 100 . Hence as many as 199 samples of the IRFs are used, equivalent to 26.5 seconds of response, or five cycles of the fundamental mode.

At the same $N$ of poles is incremented to 100 , hence for $a=b=N=100, \mathbf{H}$ is $400 \times 400$ and up to 100 pole-pairs are obtained, identifying 50 possible modes. Some of these modes have negative or very high damping or zero correlation with regenerated data and are filtered out. The numerical correlation indicator used is the extended modal amplitude coherence or 'emac' $(18,26)$. From what remains, a clear pattern of recurring and probably realistic modes emerges as the size of $\mathbf{H}$ and system order increases. Meanwhile artificial modes which appear in increasing numbers are revealed by their transience, unrealistic modal parameters and shapes and low values of emac.

In Fig. 17 the heights of the bars indicate the mode strength or msv (18) and the shading indicates the emac; white for perfect, black for zero. Clearly the winddriven fundamental modes are strongest in this response. The possibilities for graphical presentation are endless even for the same type of display e.g. keeping $a / b$ constant and increasing $N$ or vice versa, using rectangular $\mathbf{X}_{i}$, differing $a / b$ and so on.

Figure 18 plots the damping recovered by NExT/ERA applied to a vector of cross-power spectra from Office Tower $2, \mathbf{X}_{i}$ having dimension $20 \times 1$. In this exercise dimension $b$ is fixed (at 10) and $N$ is kept to a maximum of 40 as $a$ increases to 800 , covering the first 16 seconds of the impulse response function, around five cycles of the fundamental mode. Damping estimates generally decrease and stabilise around $a=300$. Further increase of $a$ tends to eliminate higher frequency modes as the slower decaying low frequency modes persist.

In these studies the number of singular values and vectors to use for reconstructing $\mathbf{A}$ is chosen manually rather than adjusted automatically according to some threshold, as there is usually a gradual rather than sudden drop in values. It is left to the user to judge which modes are realistic.

Mode shape quality indicators are available but are not always very helpful in civil structures, and the best way to judge performance is against a perfect solution. To this end a 16 degree of freedom lumped mass stick model was used to generate simulated time series of response due random and uncorrelated loading on a 16 storey building. NExT/ERA was applied directly to the time series and to the single-average cross-power spectra using only $a, b=25$ blocks of $16 \times 16$ matrices. Peak picking and IPP were used on the cross- 
powers and all were compared with the analytical solution. Table 3 shows the modal assurance criterion (MAC) value, equivalent to a correlation coefficient, between the modes and the analytical solution.

Surprisingly, despite the high variance usually quoted for mode shapes identified from frequency domain methods, errors for the lowest four modes were very small. While a restricted simulation, NExT/ERA identified a higher frequency range of modes with the lowest modes identified at least as accurately as peak picking/IPP.

With this confidence in identified mode shapes it would seem impossible to blame variance errors or problems with the identification for the ragged appearance of the modes in Figs. 14 and 15. The blame has to be with low signal to noise or some other effects to do with sensor placement at certain levels. Bear in mind that signal levels for these modes corresponded to less than $1 \mu \mathrm{g}$ RMS during periods of zero wind and the result is not so bad.

\section{CONCLUSIONS}

The performance of two 'response only' modal analysis procedures, NExT/ERA and frequency domain decomposition (or IPP) has been examined via case studies on two tall buildings. For one building the results have been compared with a previous more elaborate study and shown to reveal reliable descriptions using a range of signals caused by typical lateral loading such as a distant earthquake and local wind.

For the second building, a complete modal survey was conducted and the data analysed using the new procedures. The speed and flexibility of the NExT/ERA procedure made it possible to examine the complex modal behaviour of the structure and determine the nature of the modes. Provided all sensor calibrations and locations are known it is possible to see, on site, how mode shapes develop as more measurements are made. In parallel the simpler frequency domain decomposition procedure allowed relatively inexperienced undergraduates to identify a convincing set of lower frequency modes.

The effectiveness and accuracy of both procedures has been examined. IPP simplifies and even purifies the normal peak-picking procedure. For NExT/ERA there are a number of control parameters to adjust and the reliability of the results is to some extent indicated by numerical quality indicators, but there is a lack of the 'feel' normally found with graphical curve-fitting. However, a satisfying graphical representation of the reliability of the results can be obtained using convergence studies that show the optimal values of control parameters for reliable results while at the same time engineering judgement is used to assess whether a mode is realistic. In general the NExT/ERA procedure appears to be a very powerful tool that, subject to some constraints, works well for both ambient and forced vibration testing.

\section{ACKNOWLEDGEMENTS}


The author is grateful to Jurong Town Corporation and Yeo Gee Liek for the opportunity to test the new JTC headquarters. Students Desmond Ong, Ng Wee Boon and Goh Wei Beng carried out the testing of JTC summit. Once again the author thanks City Developments Ltd for access to Republic Plaza. The core ERA algorithms used were coded by Los Alamos National Labs, USA. 
Table 1 Natural frequencies and directions of modes for unoccupied Republic Plaza (Office Tower 1)

\begin{tabular}{|l|l|l|l|l|l|l|}
\hline \multirow{2}{*}{ mode } & \multicolumn{2}{|l|}{ A-direction } & \multicolumn{3}{l|}{ B-direction } \\
\cline { 2 - 8 } & & Frequency $/ \mathrm{Hz}$ & $\theta /^{\circ}$ & & Frequency $/ \mathrm{Hz}$ & $\theta /{ }^{\circ}$ \\
\hline 1 & A1 & 0.192 & $62: 74$ & B1 & 0.201 & $-20:-16$ \\
\hline 2 & A2 & 0.702 & $71: 76$ & B2 & 0.749 & $-16:-10$ \\
\hline 3 & A3 & 1.553 & - & B3 & 1.739 & $-7:-2$ \\
\hline 4 & A4 & 2.486 & - & B4 & 3.004 & $-6:-1$ \\
\hline
\end{tabular}


Table 2 Summary of modes for JTC Summit (Office Tower 2)

\begin{tabular}{|c|c|c|c|c|}
\hline Mode & Frequency/Hz & Mode content & Dominating & Comments \\
\hline 1 & 0.324 & $\mathrm{Y}$ & $\mathrm{Y} 1$ & \\
\hline 2 & 0.517 & $\mathrm{X} / \mathrm{T}$ & $\mathrm{T} 1$ & (also X1) \\
\hline 3 & 1.27 & $\mathrm{Y}$ & $\mathrm{Y} 2$ & \\
\hline 4 & 1.60 & $\mathrm{~T}$ & $\mathrm{~T} 2$ & \\
\hline 5 & 1.63 & $\mathrm{X} / \mathrm{T}$ & $\mathrm{X} 2$ & \\
\hline 6 & 2.70 & $\mathrm{Y} / \mathrm{T}$ & $\mathrm{Y} 3$ & Well 2 moves \\
\hline 7 & 3.03 & $\mathrm{~T} / \mathrm{X}$ & $\mathrm{T} 3$ & (also X3) \\
\hline 8 & 4.20 & $\mathrm{Y} / \mathrm{T}$ & $\mathrm{T} 4$ & Well 2 moves \\
\hline 9 & 4.70 & $\mathrm{X}$ & $\mathrm{X} 4$ & \\
\hline 10 & 5.38 & $\mathrm{X} / \mathrm{Y} / \mathrm{T}$ & $\mathrm{Y} 4$ & Well 1 moves \\
\hline 11 & 6.72 & $\mathrm{X}$ & $\mathrm{X} 5$ & \\
\hline 12 & 7.44 & $\mathrm{Y}$ & $\mathrm{Y} 5$ & \\
\hline
\end{tabular}


Table 3 MAC correlation coefficients between analytical mode shapes and first 12 modes for 16 degree of freedom simulation.

ERA1 is in time domain with cross-covariance functions

ERA2 is from frequency domain via cross-power spectra

$\mathrm{PKP}$ is from peak-picking of cross-power spectra

IPP is from first singular vector of cross-powers

- means cannot be identified due to weak/noisy signal.

\begin{tabular}{|l|l|l|l|l|l|l|l|l|l|l|l|l|}
\hline Mode: & 1 & 2 & 3 & 4 & 5 & 6 & 7 & 8 & 9 & 10 & 11 & 12 \\
\hline ERA1 & 1.0 & 1.0 & .996 & .997 & .995 & .982 & .914 & .913 & .881 & .763 & .867 & .629 \\
\hline ERA2 & 1.0 & 1.0 & .999 & 1 & .995 & .989 & .992 & .563 & .210 & .002 & .026 & .051 \\
\hline PKP & 1.0 & 1.0 & .999 & .982 & - & - & - & - & - & - & - & - \\
\hline IPP & 1.0 & .999 & .999 & .934 & - & - & - & - & - & - & - & - \\
\hline
\end{tabular}




\section{REFERENCES}

1 Brownjohn JMW, Xia PQ, 'Dynamic assessment of curved cable-stayed bridge by model updating', ASCE Journal of Structural Engineering 2000, 126(2), 252-260.

2 Brownjohn JMW, Pan TC, Deng XY, 'Macro-updating of finite element modeling for core systems of tall buildings', Proceedings, $14^{\text {th }}$ ASCE Engineering Mechanics Conference, Austin, May 2000.

3 Tamura Y, Suda K, Sasaki A, 'Damping in buildings for wind resistant design', International symposium on wind and structures for the $21^{\text {st }}$ century. 26-28 Jan 2000, Cheju, Korea.

4 Glanvill MJ, Kwok KCS, 'Dynamic characteristics and wind induced response of a steel frame tower', Journal of wind Engineering \& Industiral Aerodynamics 1995, 54/55, 133-149.

5 Brownjohn JMW, 'Vibration characteristics of a suspension footbridge', Journal of Sound and Vibration, 1997, 202(1), 29-46.

6 Hudson DE, 'Dynamic tests of full-scale structures', Earthquake Engineering, $R$ L Wiegel (Ed), 1970.

7 Galambos TV, Mayes RC, 'Lessons from dynamic tests of an eleven storey building', Engineering Structures, 19791.

8 Littler JD, 'Forced vibration tests on Sheffield University Arts Tower', Conference on Civil Engineering Dynamics, Society for Earthquake and Civil Engineering Dynamics, Bristol UK, March 1988, 61-80.

9 Williams C, Tsang WF, (1988) 'Determination of structural parameters from full-scale vibration tests' Conference on Civil Engineering Dynamics, Society for Earthquake and Civil Engineering Dynamics, Bristol UK, March 1988, 43-60.

10 Maia NMM, Silva JMM, He J, Lieven NAJ, Lin RM, Skingle GW, To W, Urgueira APV 'Theoretical and Experimental Modal Analysis', Research Studies Press Ltd, 1997.

11 Carder DS, 'Observed vibrations of bridges', Bulletin, Seismological Society of America, 1937, 27.

12 Severn RT, Jeary AP, Ellis BR, 'Forced vibration tests and theoretical studies on arch dams', Proceedings ICE pt 2 1980, 69, Sept.

13 Simiu E, Scanlan RH, Wind Effects on Structures. Wiley, New York, 1978.

14 Brownjohn JMW, Ang CK, 'full-scale dynamic response of a high rise building subject to lateral loading'. ASCE Journal of Performance of constructed Facilities 12(1), 1998, 33-40.

15 Smith JW, Vibration of structures: applications tin civil engineering design. Chapman \& Hall London, 1988.

16 Welch PD, 'The use of fast Fourier transform for the estimation of power spectra: A method based on time averaging over short, modified periodograms', IEEE Transactions, 1967, AU-15, 70-73.

17 Brownjohn JMW, 'Estimation of damping in suspension bridges', Structures and Buildings, Proceedings, Institution of Civil Engineers, 1994 104, UK, 401-415.

18 James GH, Carne TG, Lauffer JP, 'The natural excitation technique (NExT) for modal parameter extraction from operating structures', Journal of Analytical and Experimental Modal Analysis 1995, 10 (2), 260-277. 
19 Juang J-N, Pappa RS, 'An eigensystem realisation algorithm for modal parameter identification and model reduction', AIAA Journal of Guidance, 1985, 8(5), 620-627.

20 http://sdb-www.larc.nasa.gov/SDB/Research/data/ERA_biblio.html

21 Juang J N, 'Applied system realisation', Prentice Hall, 1994.

22 Brinker R, Zhang L, Andersen $P$ (2000) 'Modal identification from ambient responses using frequency domain decomposition' Proceedings, IMAC 18 Feb 7-10 San Antonio, 625-630.

23 Brownjohn JMW, Hao H, Pan TC, 'Assessment of structural condition of bridges by dynamic measurements' Applied Research Project Report RP5/97, NTU School of CEE, Singapore, April 2001.

24 Brownjohn JMW, Pan TC, Deng XY, (2000) 'Correlating dynamic characteristics from field measurements and numerical analysis of high-rise buildings', Earthquake Engineering and Structural Dynamics 29, 523-543.

25 http://ext.lanl.gov/projects/damage_id/

26 Pappa RS, Elliott KB, Schenk A, 'Consistent-mode indicator for the eigensystem realisation algorithm' AIAA Journal of Guidance and Dynamics 1993, 16(5), 852-858. 


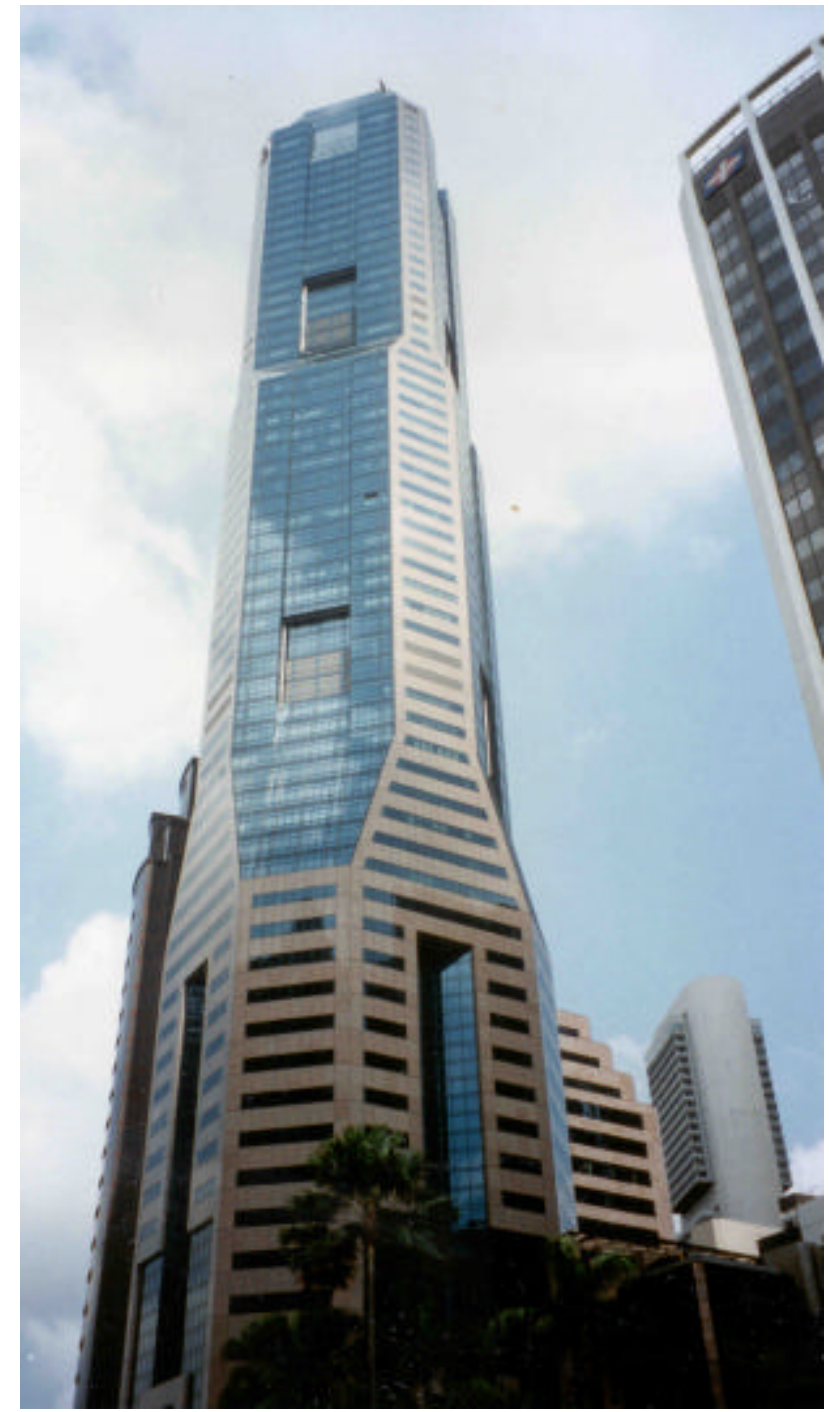

Figure 1 Office Tower 1: Republic Plaza. 
Figure 2 Plan on roof level for Republic Plaza showing principal directions A, B

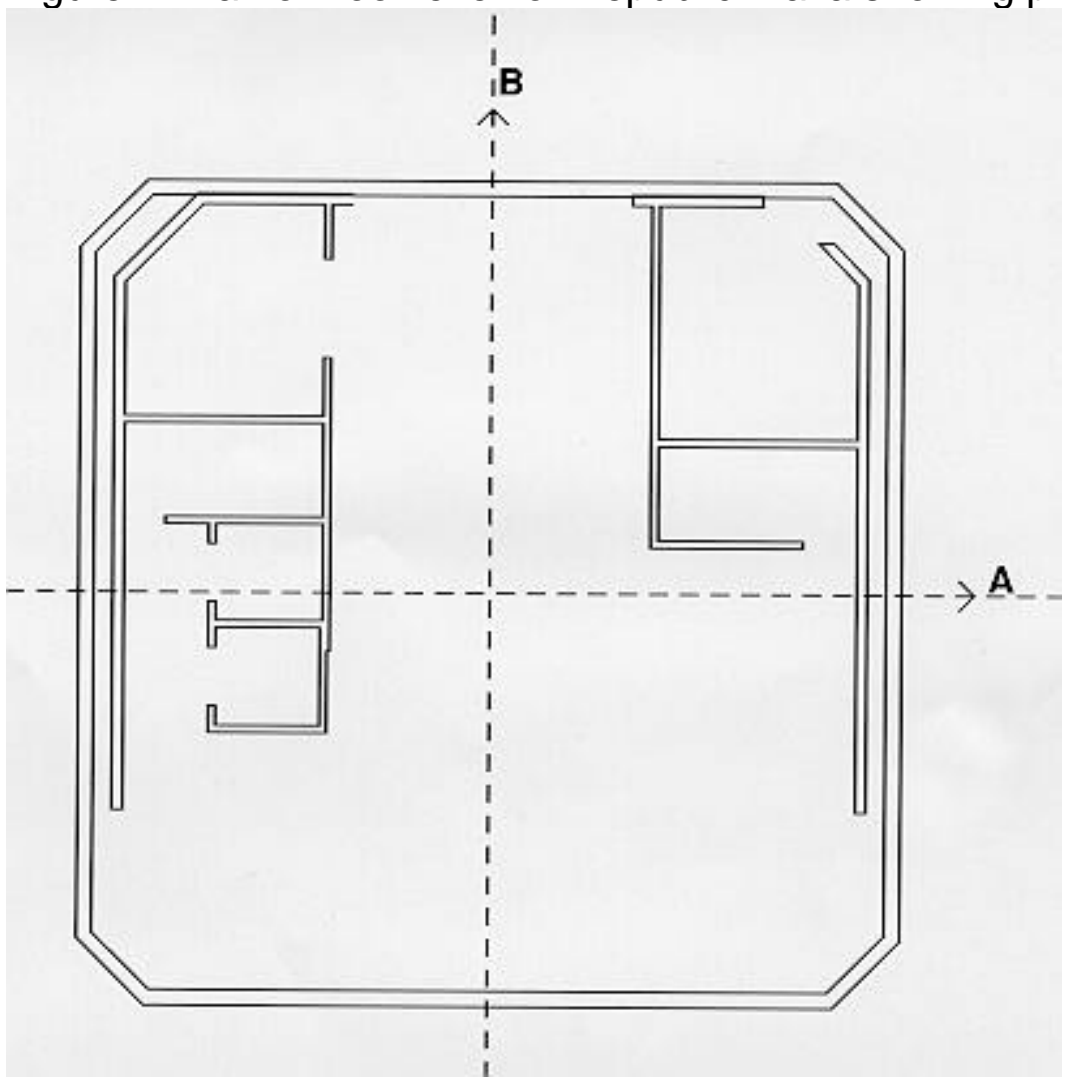


Figure 3 Office Tower 2: JTC summit building. Curved façade visible at roof levels covers quadrant of building invisible from camera.

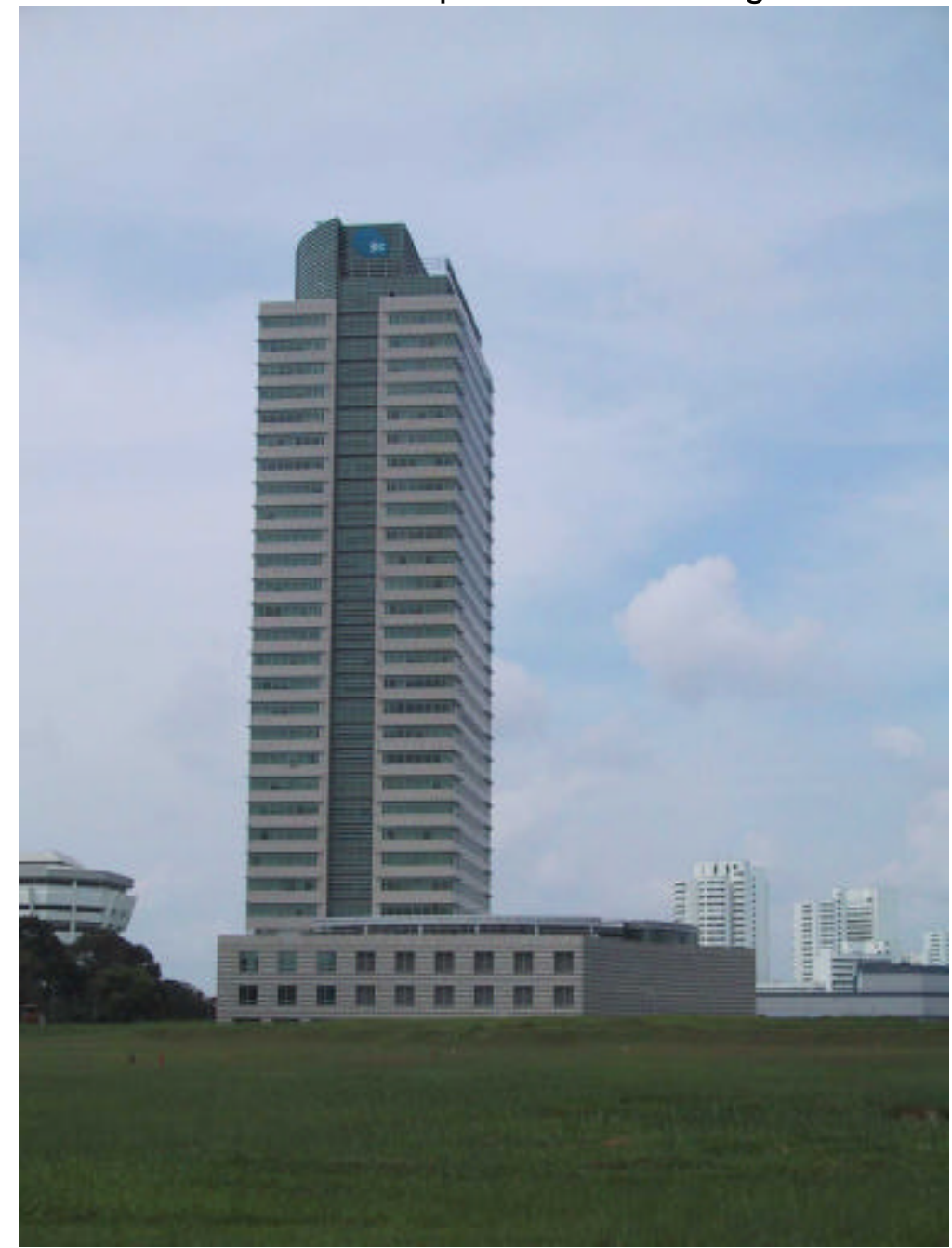




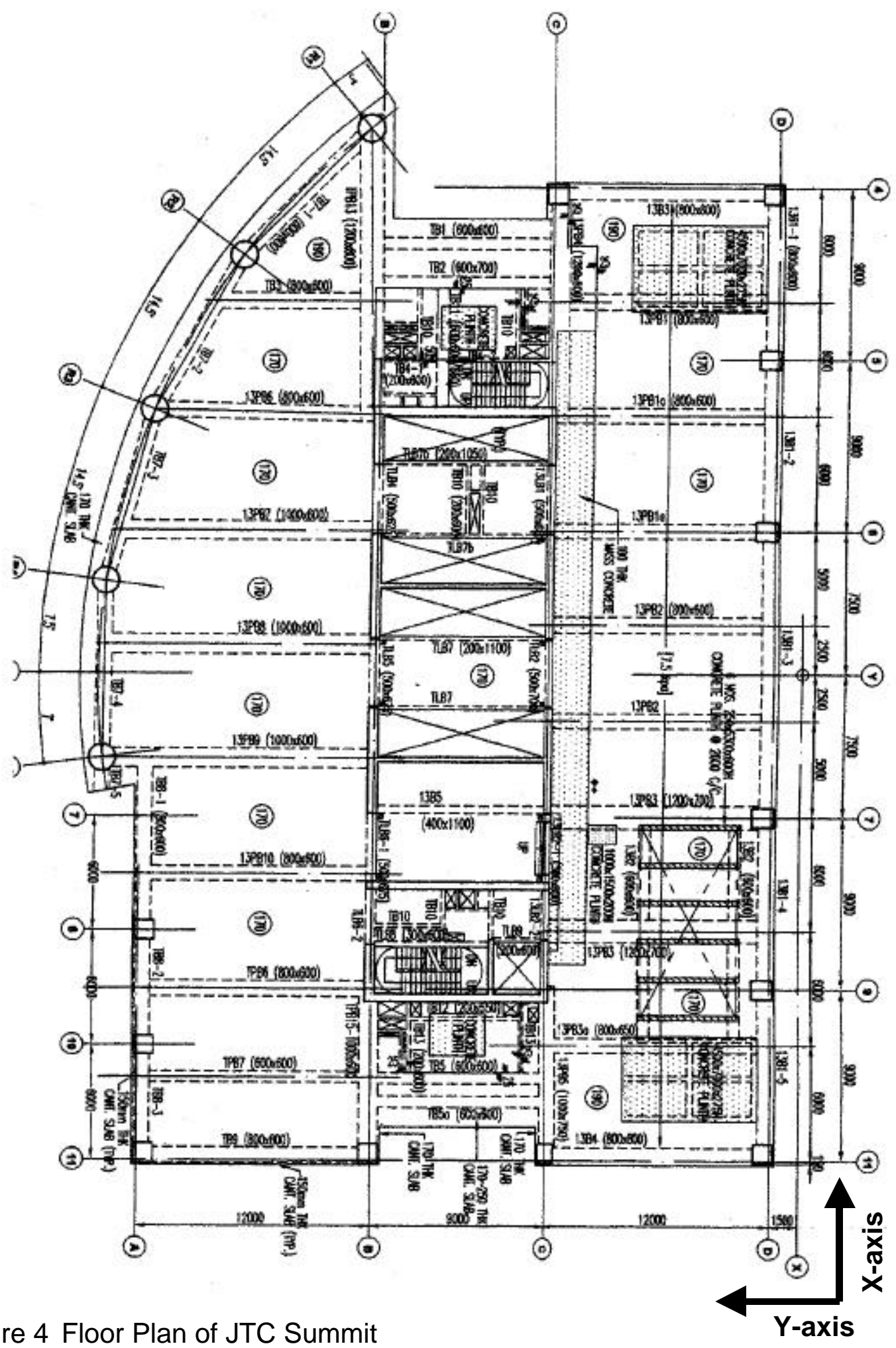

Figure 4 Floor Plan of JTC Summit 
Figure 5 Office Tower 1: Time series and spectrogram of building response to tremor.

Channel 1 is basement motion, channel 3 is motion at high storey. Units are $\mathrm{m} . \mathrm{sec}^{-2}$.
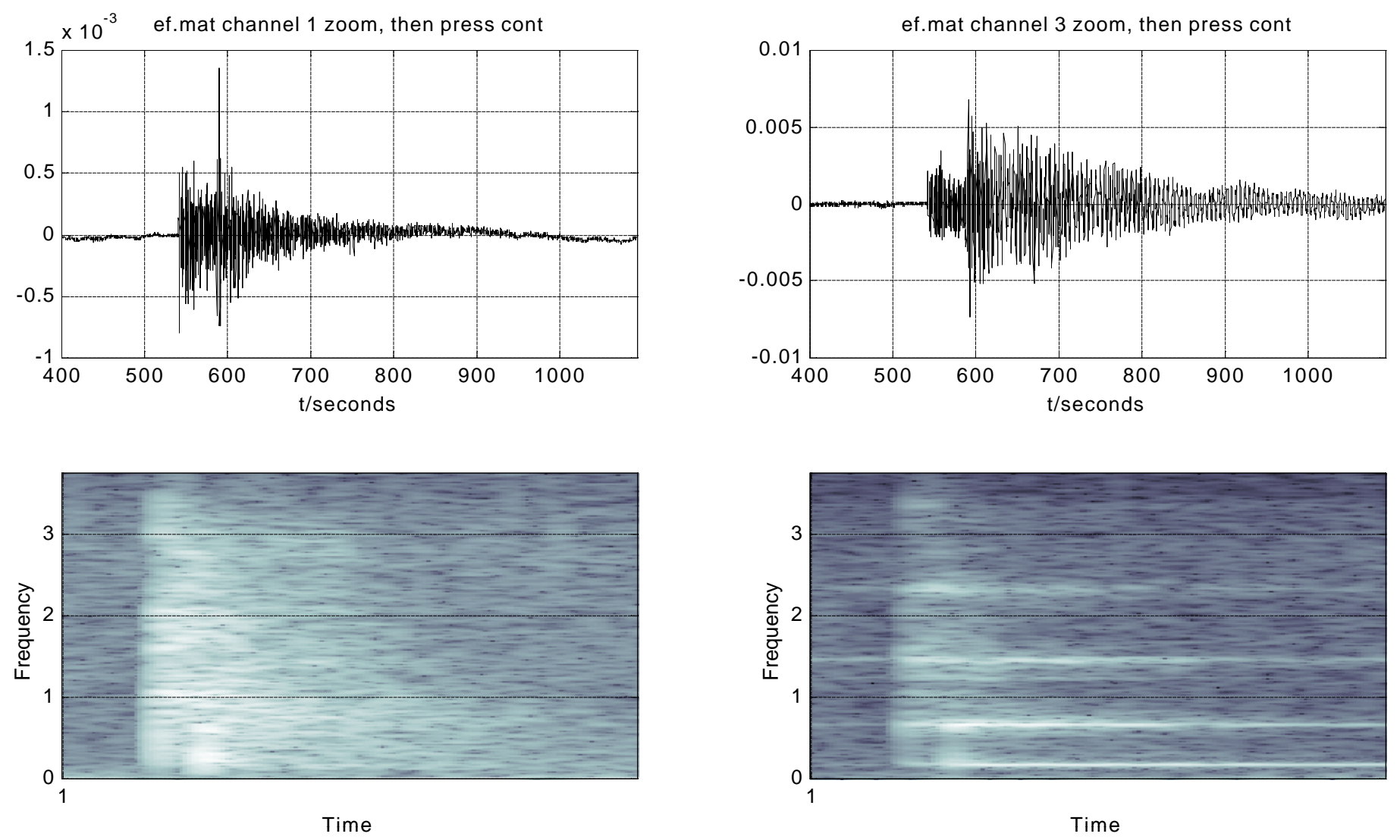
Figure 6 Office Tower 1: Cross-covariance function between basement and level 65
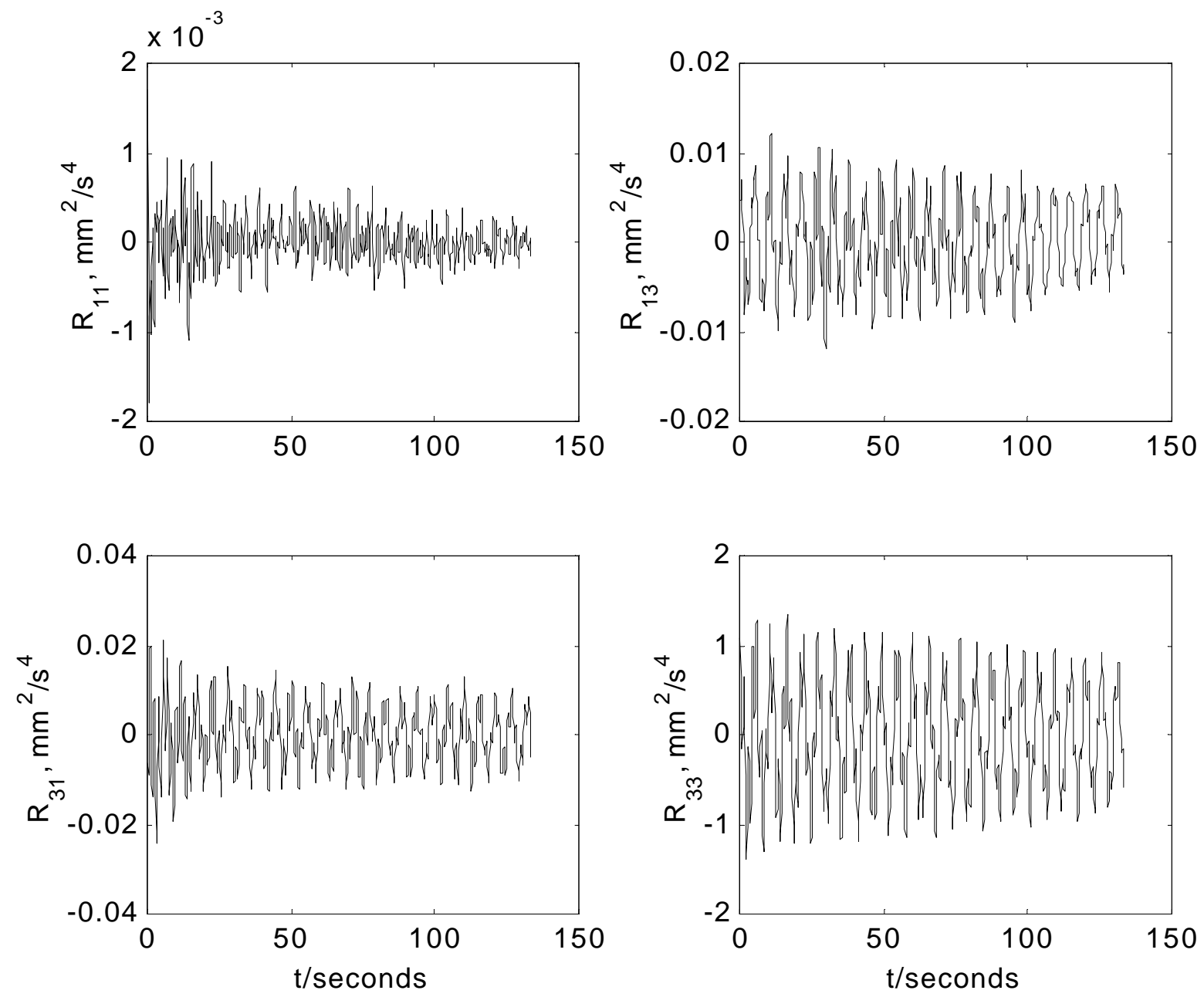
Figure 7 Office Tower 1: Transfer function between basement and building. Left frequency domain, right time domain (inverse FFT)
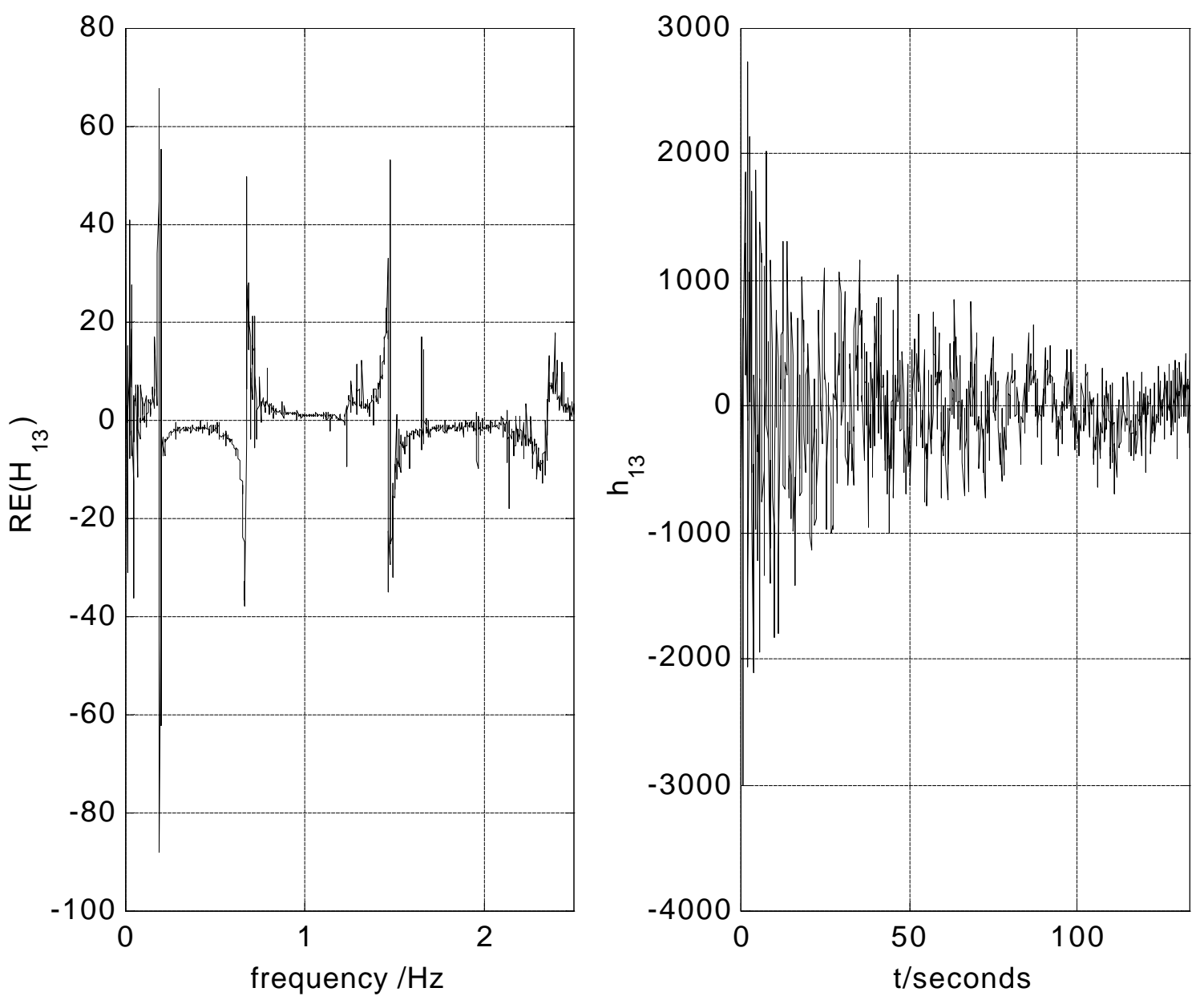
Figure 8 Office Tower 1: Auto power spectra in A and B directions obtained with fine resolution
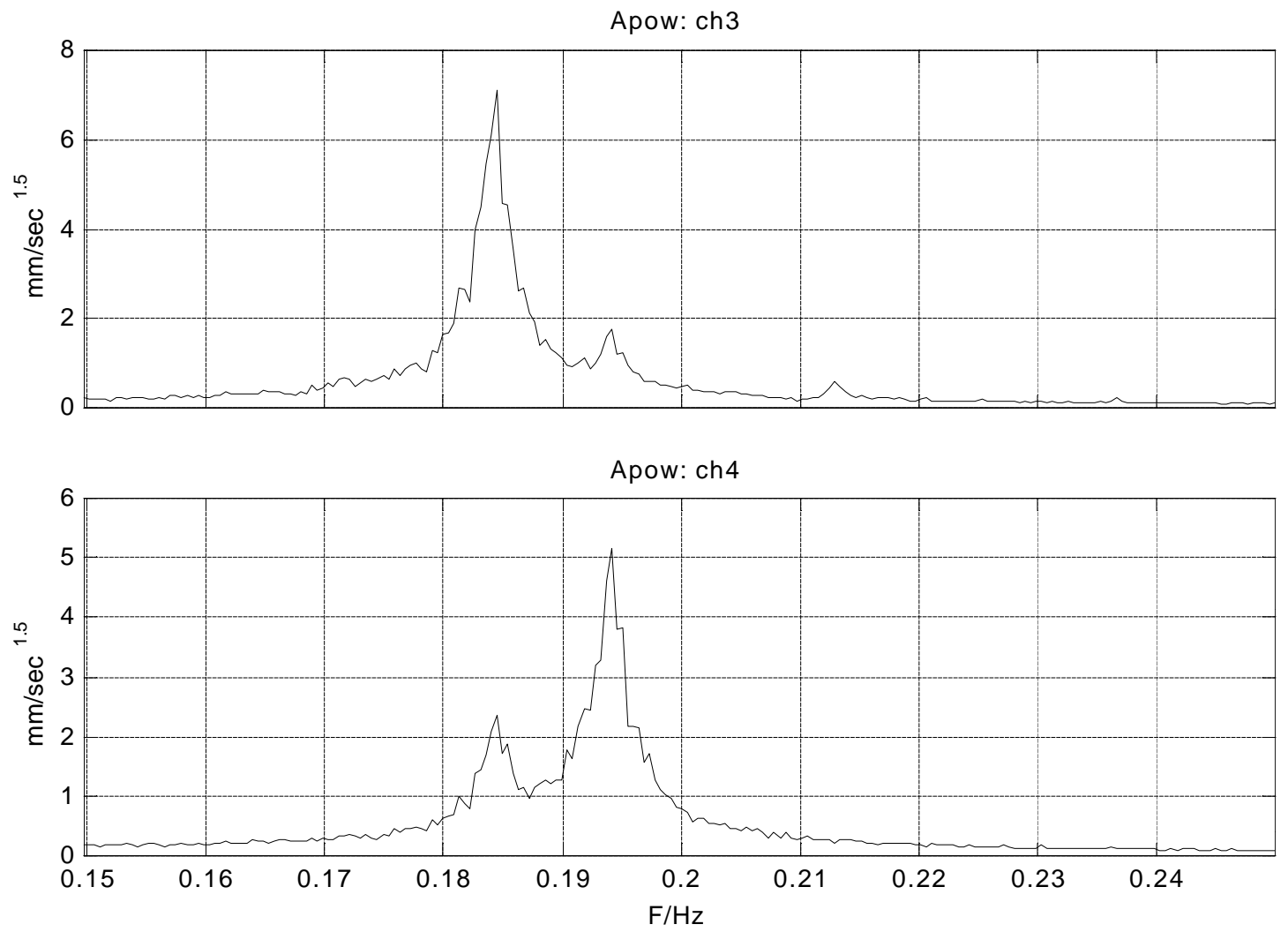
Figure 9 Office Tower 1: First and second singular vector from auto powers of Figure 8.
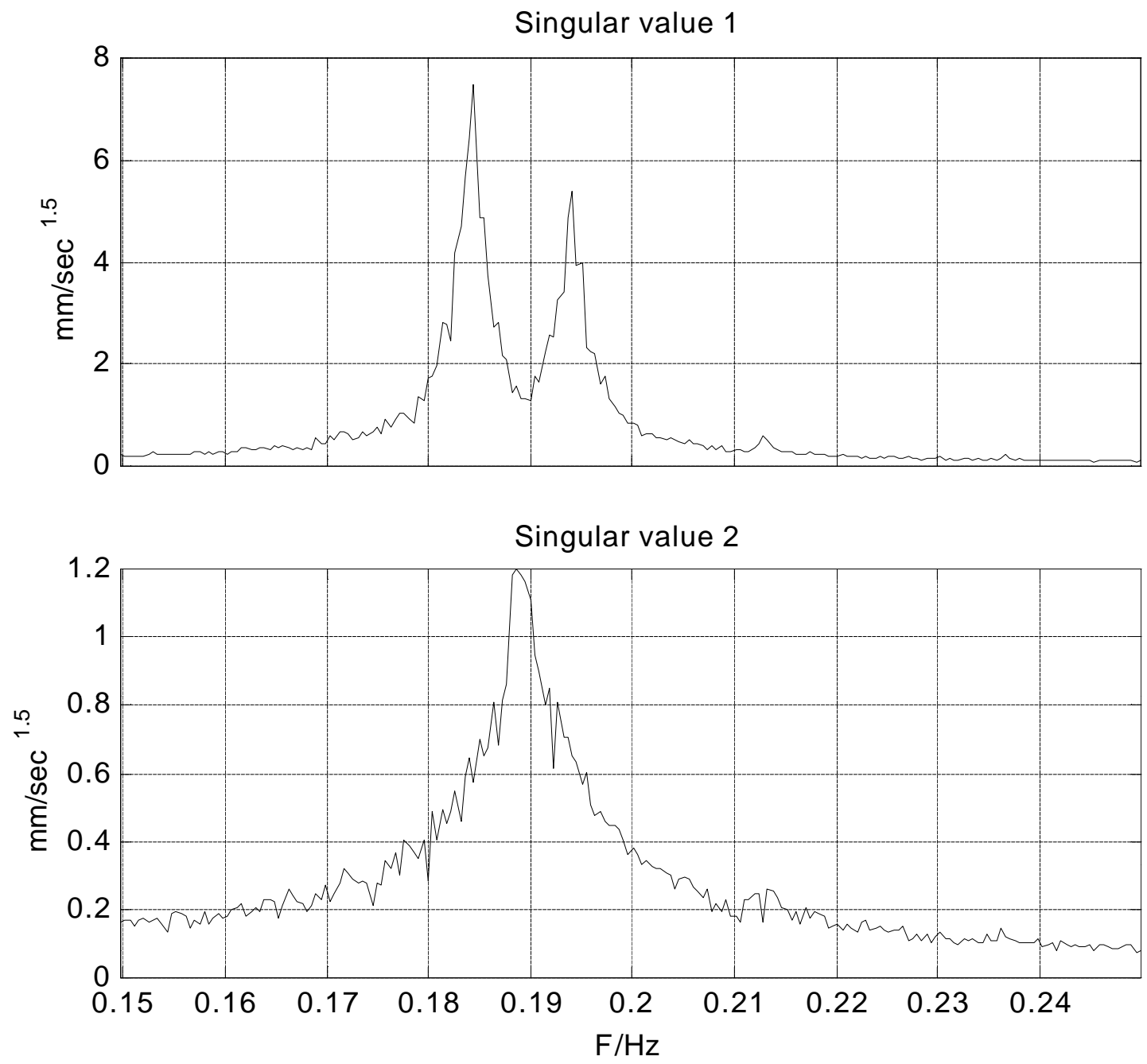
Figure 10 Office Tower 1: Auto-power spectra averaged of strong response: Upper view is basement response in orthogonal directions ' $A$ ' and ' $B$ ' Lower view is response at level 65 in $A$ and $B$ directions
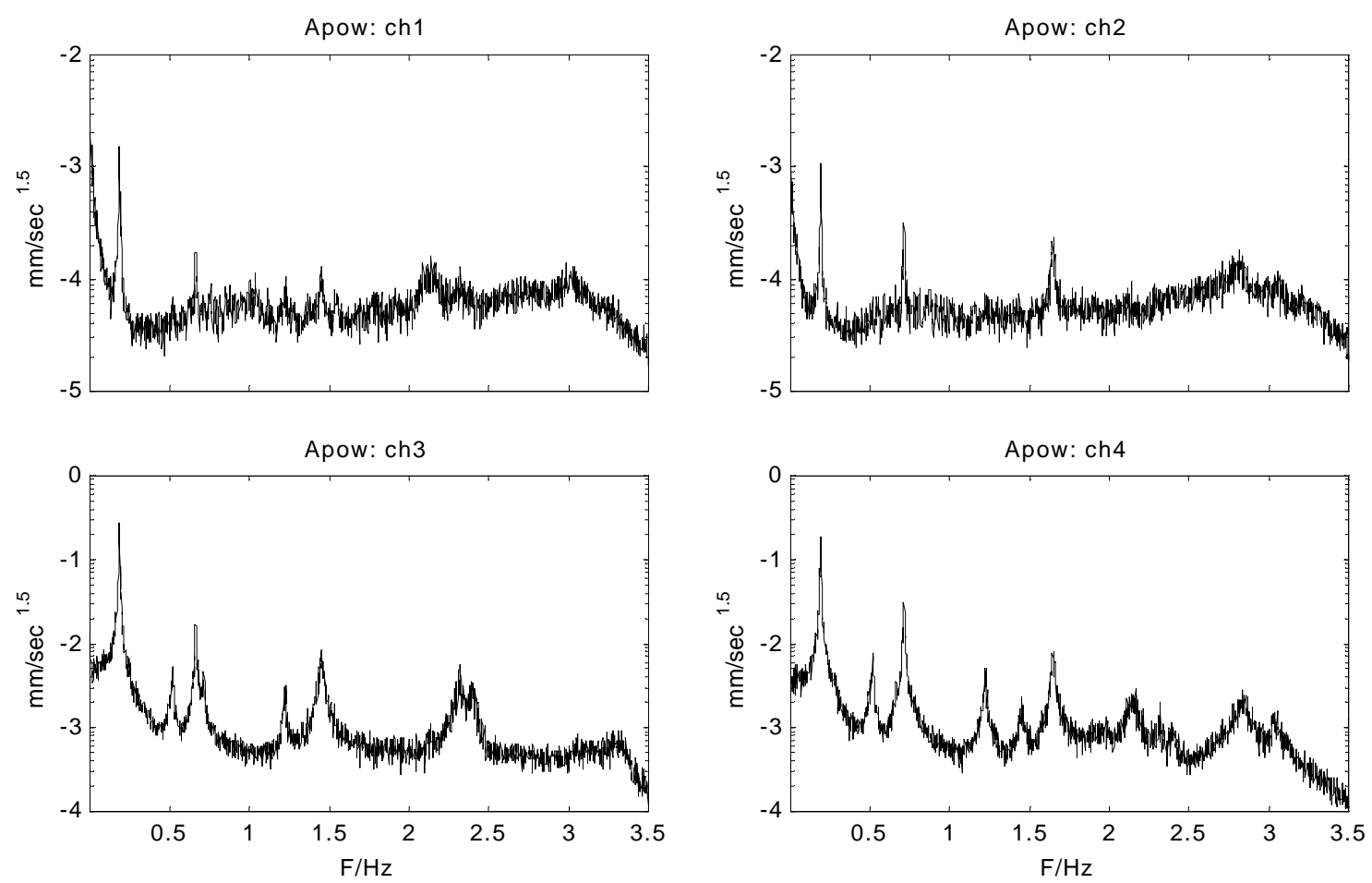
Figure 11 Office Tower 1: Mode shapes vectors at level 65 showing alignment of modes with non-principal axes A,B of Figure 2.

mode: $1 \mathrm{f}=0.1837 \mathrm{~Hz}$
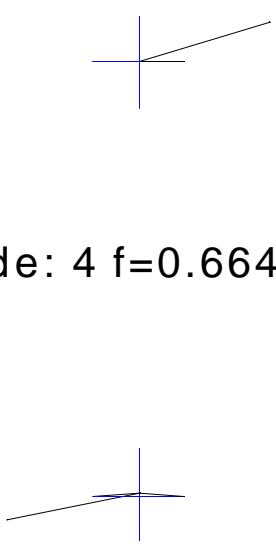

mode: $7 \mathrm{f}=1.45 \mathrm{~Hz}$

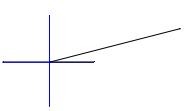

mode: $2 \mathrm{f}=0.1932 \mathrm{~Hz}$

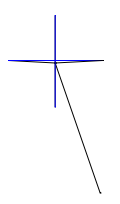

mode: $5 \mathrm{f}=0.7146 \mathrm{~Hz}$

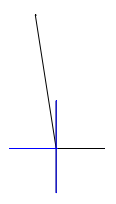

mode: $8 \mathrm{f}=1.648 \mathrm{~Hz}$

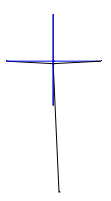

mode: $3 \mathrm{f}=0.518 \mathrm{~Hz}$

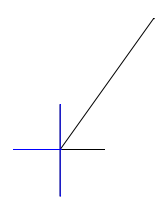

mode: $6 \mathrm{f}=1.223 \mathrm{~Hz}$

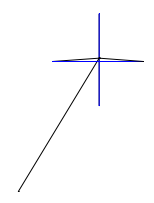

mode: $10 \mathrm{f}=2.401 \mathrm{~Hz}$ 
Figure 12 Office Tower 2: Grid of measurement points

$\begin{array}{ll}35 & 70 \\ 34 & 69 \\ 33 & 68 \\ 32 & 67 \\ 31 & 66 \\ 30 & 65 \\ 29 & 64 \\ 28 & 63 \\ 27 & 62 \\ 26 & 61 \\ 25 & 60 \\ 24 & 59 \\ 23 & 58 \\ 22 & 57 \\ 21 & 56 \\ 20 & 55 \\ 19 & 54 \\ 18 & 53 \\ 17 & 52 \\ 16 & 51 \\ 15 & 50 \\ 14 & 49 \\ 13 & 48 \\ 12 & 47 \\ 11 & 46 \\ 10 & 45 \\ 9 & 44 \\ 8 & 43 \\ 7 & 42 \\ 6 & 41 \\ 5 & 40 \\ 4 & 39 \\ 3 & 38 \\ 2 & 37 \\ 1 & 36\end{array}$


Figure 13 Office Tower 2: Auto-power spectra for JTC summit in X and $Y$ directions at level 31
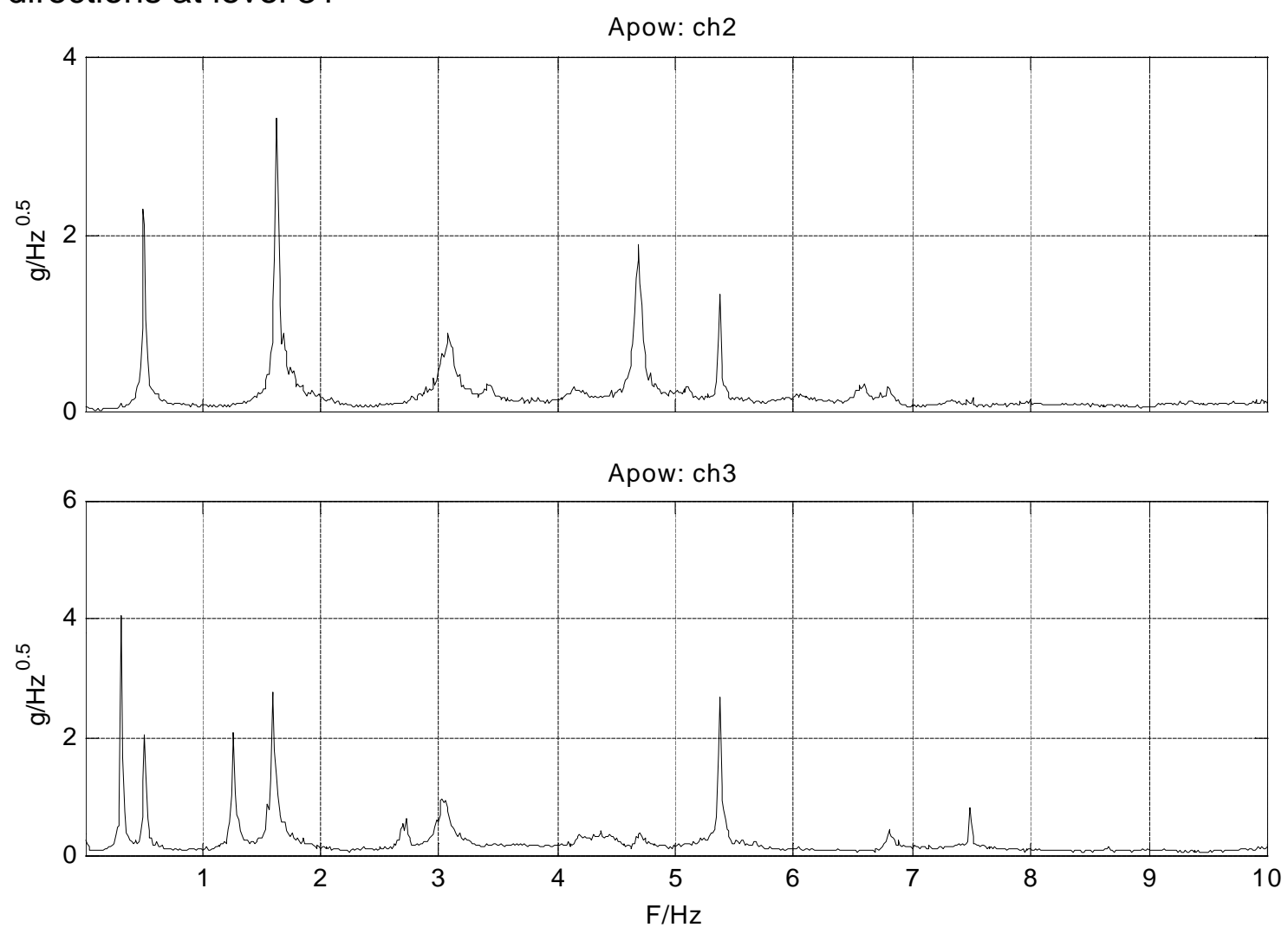
Figure $14 \quad$ Office Tower 2: Lateral $(\mathrm{Y})$ component of modes

mode: $1 \mathrm{f}=0.324 \mathrm{~Hz}$ mode: $2 \mathrm{f}=0.517 \mathrm{~Hz}$ mode: $3 \mathrm{f}=1.27 \mathrm{~Hz}$ mode: $4 \mathrm{f}=1.6 \mathrm{~Hz}$
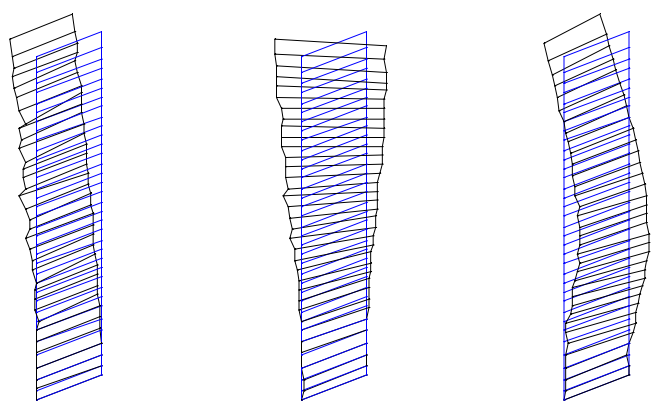

mode: $7 \mathrm{f}=3.05 \mathrm{~Hz}$ mode: $8 \mathrm{f}=4.17 \mathrm{~Hz}$
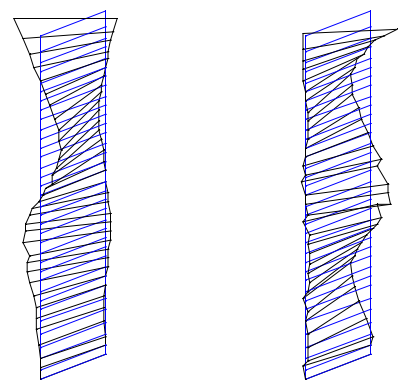

mode: $6 \mathrm{f}=2.7 \mathrm{~Hz}$

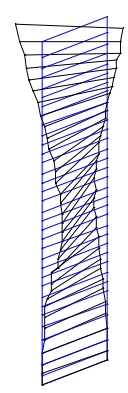

mode: $10 \mathrm{f}=5.38 \mathrm{~Hz}$

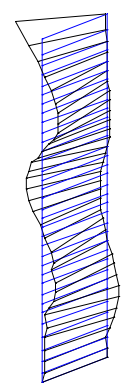

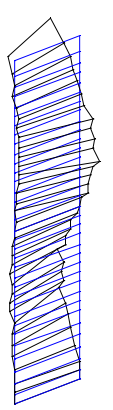

mode: $12 \mathrm{f}=7.44 \mathrm{~Hz}$

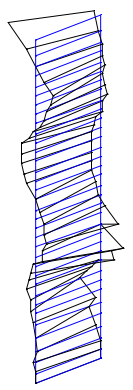


Figure 15 Office Tower 2: Longitudinal $(X)$ component of modes mode: $2 \mathrm{f}=0.517 \mathrm{~Hz}$ mode: $5 \mathrm{f}=1.63 \mathrm{~Hz}$
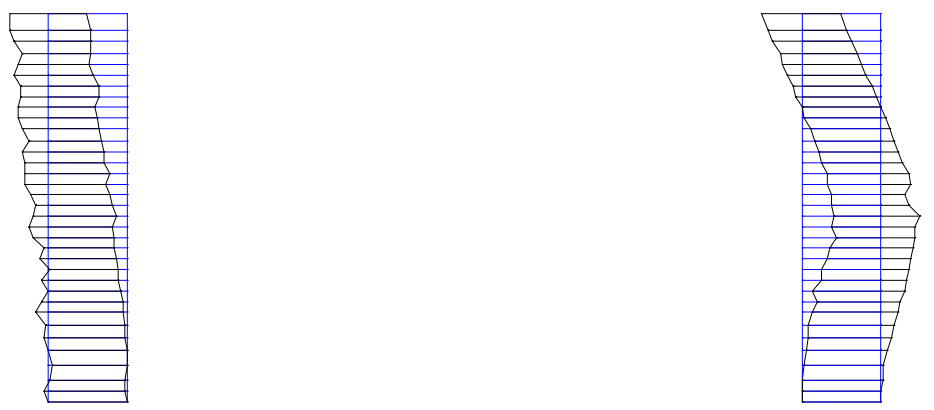

mode: $7 \mathrm{f}=3.05 \mathrm{~Hz}$

mode: $9 \mathrm{f}=4.7 \mathrm{~Hz}$

mode: $10 \mathrm{f}=5.38 \mathrm{~Hz}$ mode: $11 \mathrm{f}=6.72 \mathrm{~Hz}$
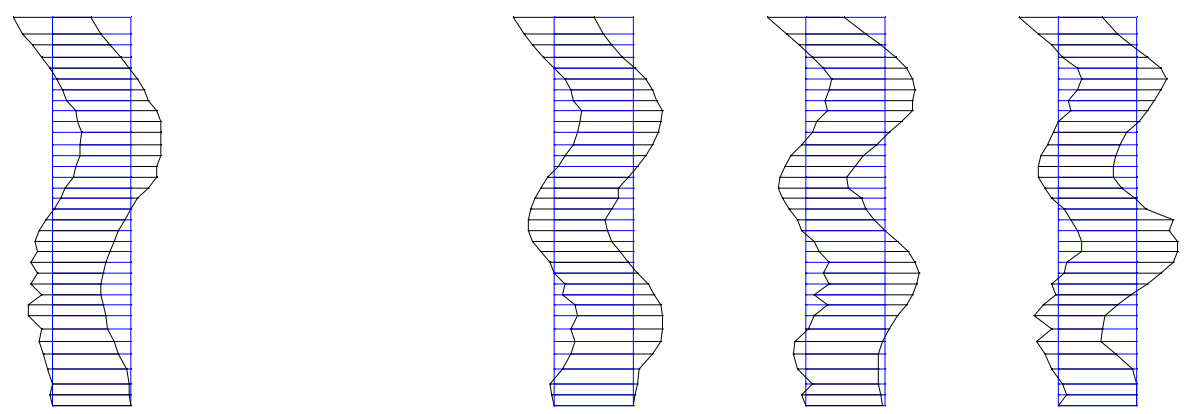
Figure 16 Office Tower 2: Plan on modes at storey 31
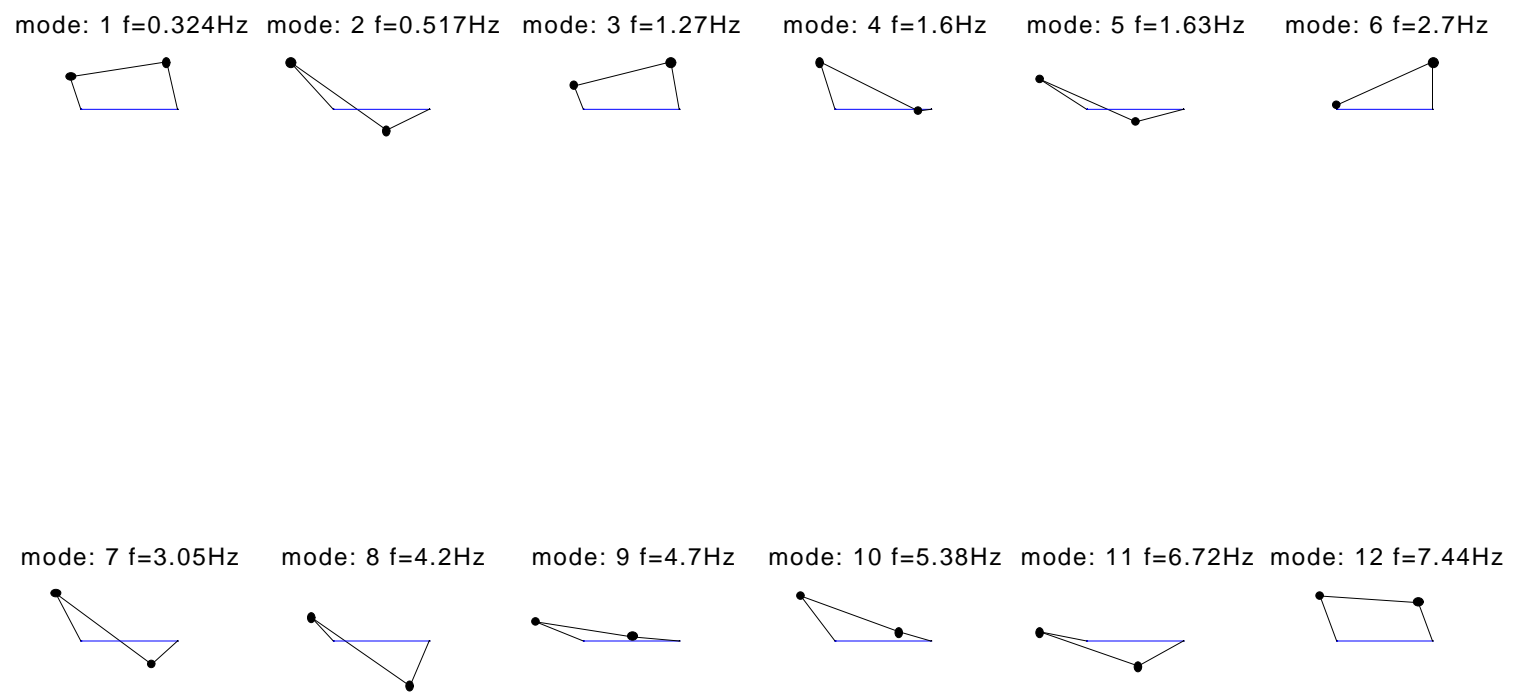


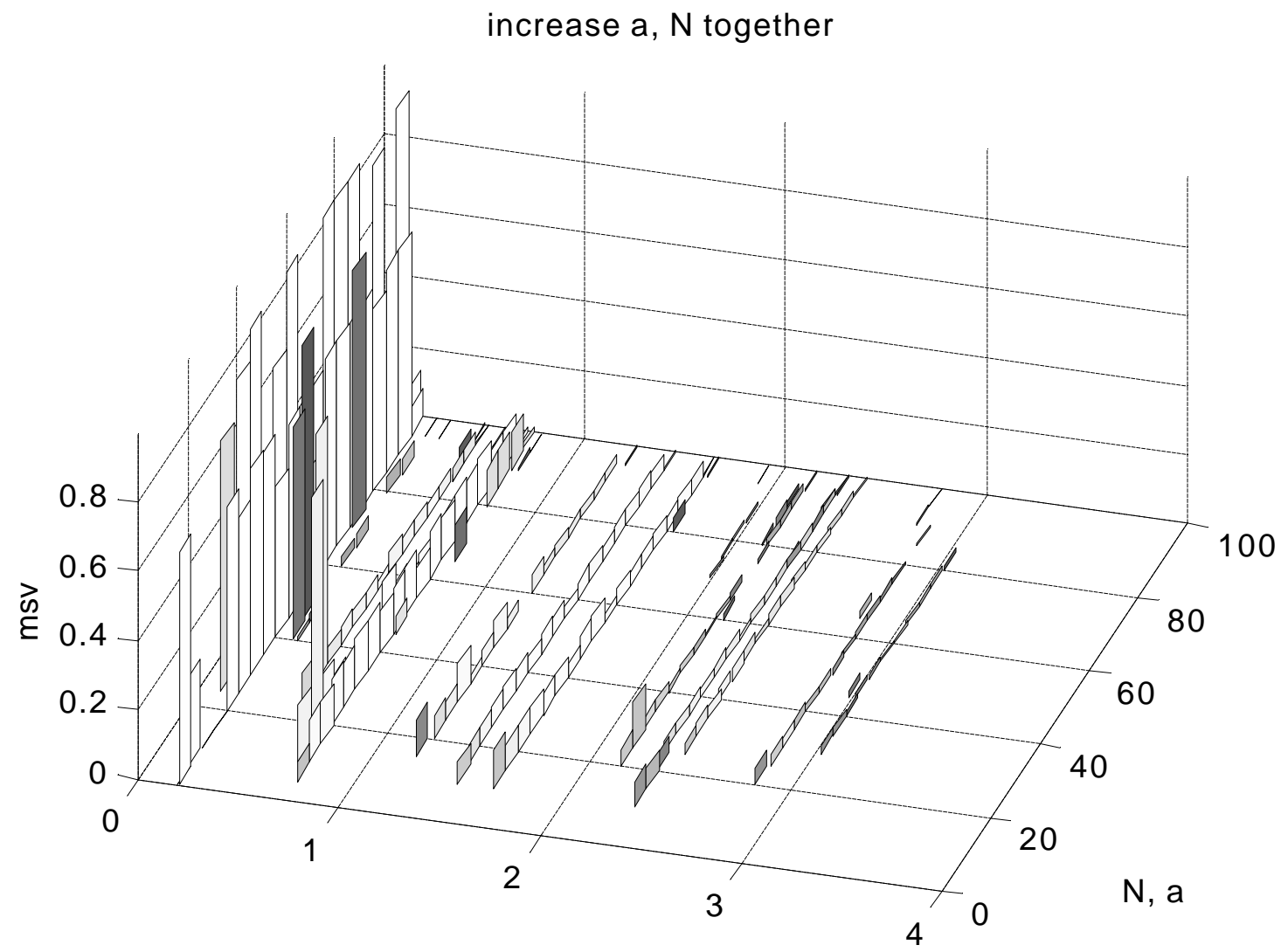

Frequency $/ \mathrm{Hz}$

Figure 17 Convergence study on data of Figure 10, increasing size of Hankel matrix $\mathbf{H}$ in steps of 5 blocks. Bar height represents mode strength, shade represents quality of mode (emac) 
Figure 18 Convergence study on damping for Office Tower 2 using data of figure 13 , increasing size of $\mathbf{H}$.
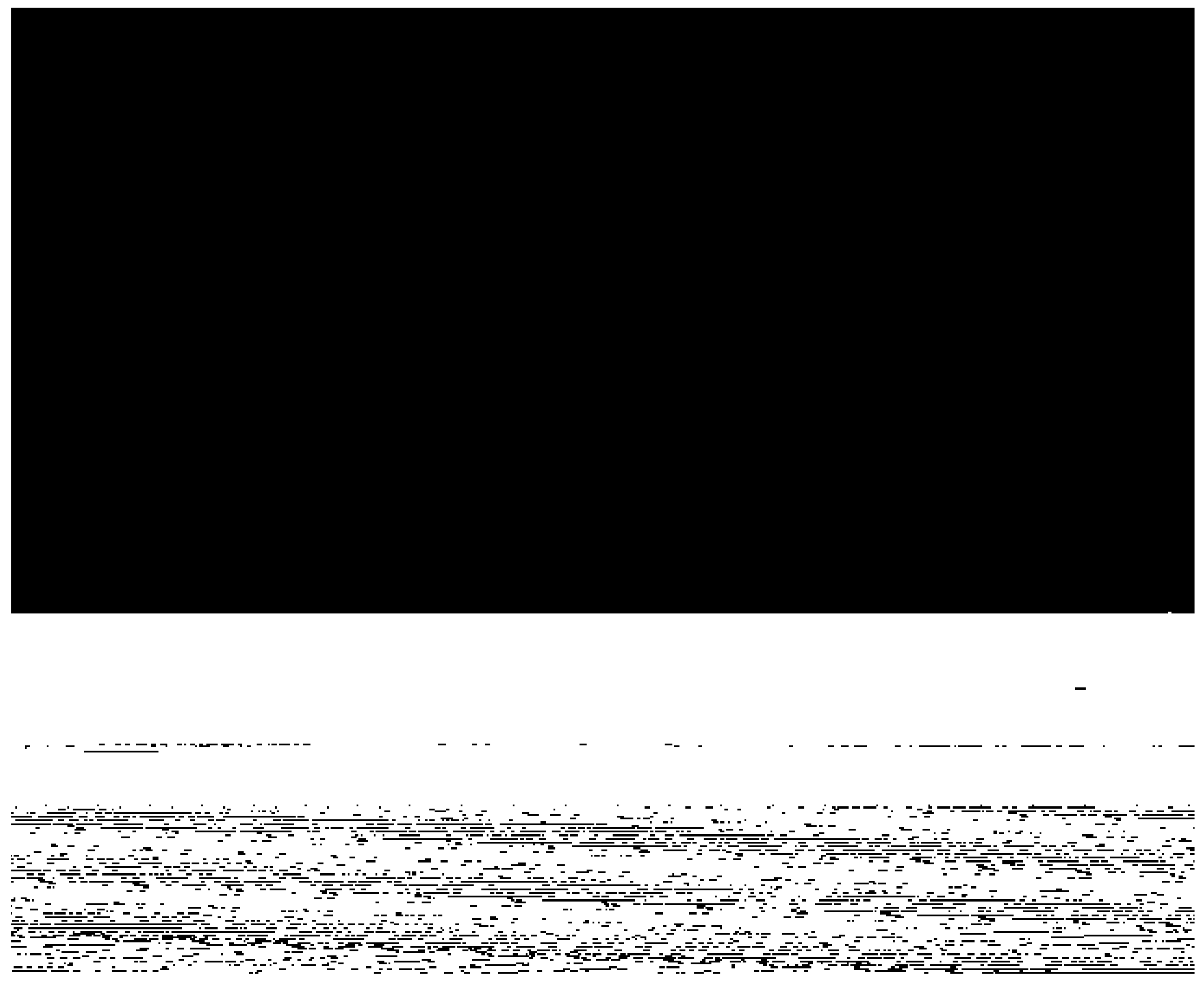